\title{
Disconnection-mediated twin junction migration mechanism in FCC metals
}

\author{
Thomas Kaufman ${ }^{1}$, Kongtao $\mathrm{Chen}^{2}$, Jian $\mathrm{Han}^{3}$, Fan $\mathrm{Cao}^{4}$, Mingjie Xu ${ }^{5}$, Fan $\mathrm{Ye}^{6}$, David Srolovitz ${ }^{3}$ and Xiaoqing
} $\operatorname{Pan}^{7}$

${ }^{1}$ University of California, Irvine, United States, ${ }^{2}$ University of Pennsylvania, United States, ${ }^{3}$ City University of Hong Kong, United States, ${ }^{4}$ Wuhan University, United States, ${ }^{5}$ Irvine Materials Research Institute, United States, ${ }^{6} \mathrm{Xi}$ An Jiao Tong University, United States, ${ }^{7}$ Department of Physics and Astronomy, University of California, Irvine, CA 92697, Irvine, California, United States

The evolution of grain boundary (GB) networks in polycrystals during thermomechanical processing is rate limited by the motion of triple junctions (TJs) [1]. Migration of GBs, and therefore TJs, occurs by nucleation and migration of disconnections (line defects with both burgers vector and step height character which generate curvature on GBs) along GBs [2]. In order to resolve the atomic structure of GBs in TEM, the boundary must be parallel to the electron beam. Therefore, resolving the atomic structure of a TJ and its constituent GBs provides a technical challenge, as all boundaries must be parallel to the electron beam simultaneously. In facecentered cubic metals, TJs where coherent twin boundaries intersect provide a model system for TEM studies, as all three grains share a common [110] zone axis [3]. The fixed orientation of coherent twin boundaries limits the set of TJs of twins (twin junctions) which may form; the two most common are the $\beta$-type and $\delta$-type $\Sigma 3$ $\Sigma 3-\Sigma 9$ TJs, where the two $\Sigma 3$ GBs meet angles of $70^{\circ}$ and $107^{\circ}$, respectively. In this work, migration of a GB network in $\mathrm{Cu}$ consisting of one $\delta$-type and one $\beta$-type twin junction was induced by heating to $300^{\circ} \mathrm{C}$ during in situ HRTEM observation. Ultrafast disconnection nucleation and migration events not able to be directly observed in situ were investigated by molecular dynamics (MD) simulations and disconnection theory.

Heating of the sample lead to migration of the GBs and TJs, as shown in Figure 1. The activation of migration mechanisms must lead to reduction of the system's energy. The $\delta$-type twin junction, and therefore also Twin 3, are observed to migrate by 9 (111) layers in Figure $1 \mathrm{~b}$ and then 6 (111) layers in Figure 1d. This leads to elimination of all GBs initially present except Twin 4, most importantly eliminating the $\Sigma 9 \mathrm{~GB}$, which has a very high energy compared to the coherent twins $\left(1 \mathrm{~J} / \mathrm{m}^{2}\right.$ for $\Sigma 9$ versus $20 \mathrm{~mJ} / \mathrm{m}^{2}$ for coherent twins). Disconnection theory predicts two disconnection-mediated migration mechanisms for the $\delta$-type twin junction in face-centered cubic metals: (1) three-layer thick pure steps nucleated in TJs onto the coherent twin under chemical potential difference; (2) partial dislocations nucleated in TJs and edges onto the coherent twin under shear stress. MD simulations on $\mathrm{Cu}$ verified mechanism (1) to occur under a synthetic chemical potential jump across the coherent twin of $0.01 \mathrm{eV} /$ atom, and mechanism (2) to occur under a constant strain rate of $\varepsilon^{\prime}=5 \times$ $10^{9} / \mathrm{s}$ applied in the plane of the $\Sigma 9$ GB. Mechanism (1) is shown in Figure 2 . The experiments verify mechanism (1): because no external stresses are applied during the in situ experiment, and no strain is observed to accumulate in similar networks near the edge of the film, the pure-step mechanism must be responsible for the dynamical phenomenon observed. The pure-step disconnections have no burgers vector, and therefore can only be driven by a chemical potential difference or curvature (and not by stress), and do not lead to accumulation of strain.

This work was supported by the Army Research Office (ARO) under Grant W911NF-19-1-0263. The authors acknowledge the use of facilities and instrumentation at the UC Irvine Materials Research Institute (IMRI) supported in part by the National Science Foundation through the Materials Research Science and Engineering Center program (DMR-2011967). 

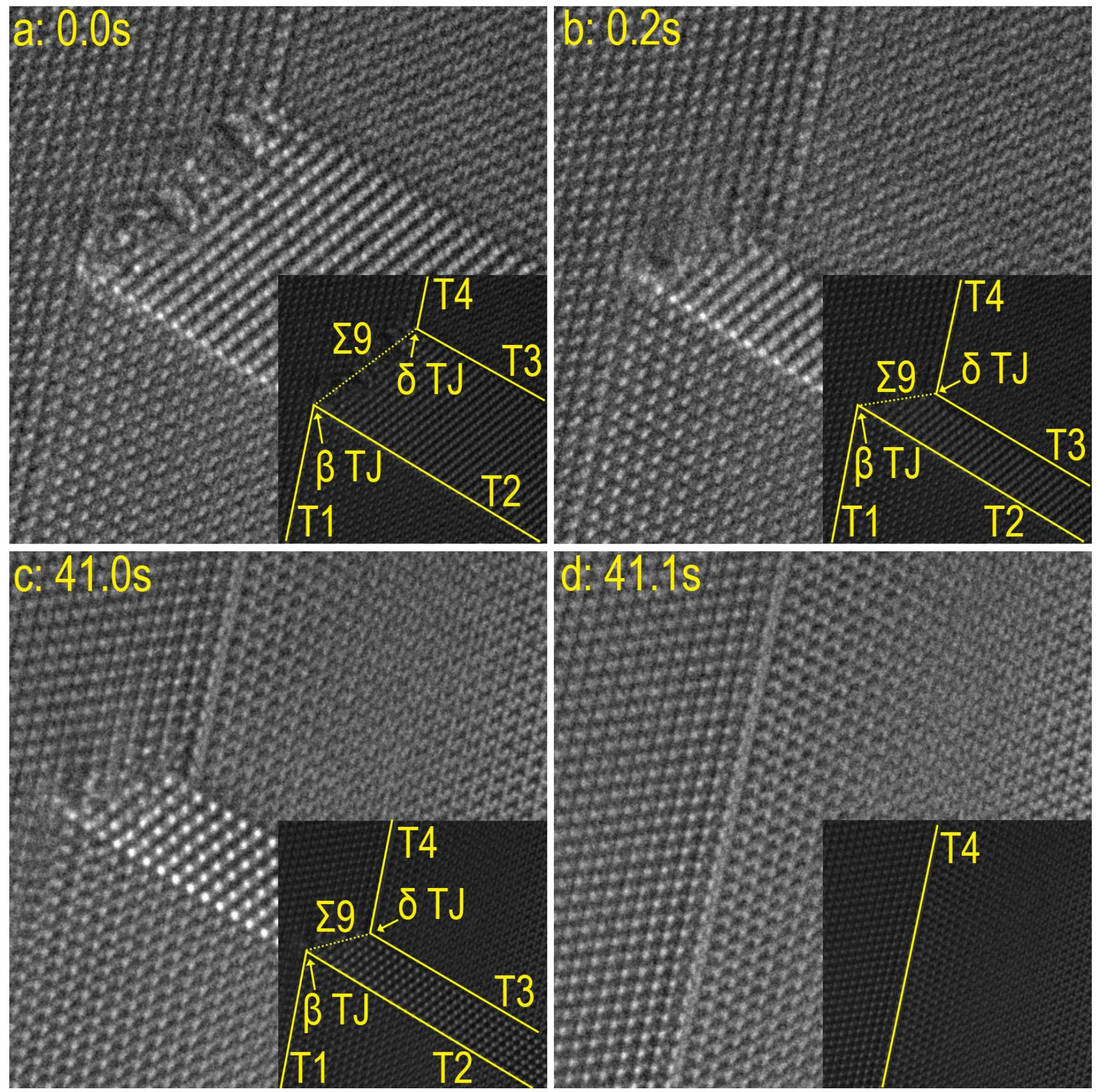

Figure 1. Figure 1: HRTEM images of a GB network in $\mathrm{Cu}$ during in situ heating at $300^{\circ} \mathrm{C}$. Elimination of twins 1,2 , and 3 occurs by migration of the $\delta$-type twin junction towards the $\beta$-type, always occurring by multiples of three (111) layers. (a) The initial state of the GB network, where twin 2 and twin 3 are separated by 15 (111) layers. (b) Migration of the $\delta$-type twin junction reduces the thickness of the twinned region from 15 to 6 (111) layers. (c) The GB network is stable for 40.8s. (d) Finally, the twinned region is eliminated, with only twin 4 persisting. 


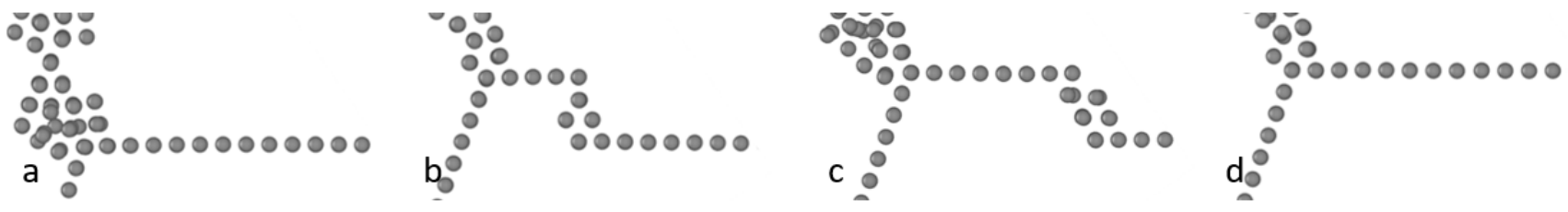

Figure 2. Figure 2: Snapshots of MD simulations on $\delta$-type twin junction in $\mathrm{Cu}$. (a) The initial state of the twin junction. (b) Nucleation of a pure-step disconnection with $b=0$ and $h=3 \times d 111$ from the $\delta$-type twin junction. (c) Migration of a pure-step disconnection along the coherent twin. (d) After the pure-step disconnection has migrated to the edge, the $\delta$-type twin junction and the horizontal coherent twin boundary migrate by three (111) layers.

\section{References}

[1] S.L. Thomas, et al., PNAS116 (2019), 8756-8765

[2] Q. Zhu, et al., Nat. Commun.10 (2019), 1-8

[3] S.L. Thomas, et al., Acta Mater.113 (2016), 301-310 